\title{
X-ray Microscopy: The Cornerstone for Correlative Characterization Methods in Materials Research and Life Science
}

\author{
Arno P. Merkle ${ }^{1}$, Jeff Gelb ${ }^{1}$, Alexander Orchowski ${ }^{2}$ and Jochen Fuchs ${ }^{3}$ \\ 1. Carl Zeiss X-ray Microscopy, Inc., Pleasanton, CA USA \\ 2. Carl Zeiss Microscopy GmbH, Oberkochen, Germany \\ 3. Carl Zeiss AG, Oberkochen, Germany
}

X-ray tomography has emerged as a powerful imaging technique that obtains 3D structural information from opaque samples under a variety of conditions and environments [1,2]. It has rapidly become an accepted laboratory technique offering quantitative information in both the materials sciences and life sciences. Here we present ways in which non-destructive 3D volumetric information, obtained via laboratory nanoscale and sub-micron X-ray microscopy (XRM) are increasingly used to probe scientific questions as a complement to Electron- and Light-based microscopy methods. These correlative methods, relating to XRM, provide an opportunity to study materials evolution at multiple length scales in $3 \mathrm{D}$ and utilize this information to inform or guide postmortem analysis to be most efficient.

In materials research, the motivation to correlate XRM information with postmortem EM (SEM, FIBSEM or TEM) stems from three primary reasons. First, this workflow is used to complement time dependent materials evolution (4D) studies with higher resolution imaging, diffraction or spectroscopic information. Second, hierarchical porous materials such as membranes or porous rock naturally exhibit features from $\mathrm{mm} \rightarrow \mathrm{nm}$, all which require characterization of a single volume in $3 \mathrm{D}$ with multiple imaging modalities to define performance. Finally, XRM is used as a 3D navigation system ('Google Earth' in 3D) for targeting and finding specific buried structures of interest for extraction or cross sectional imaging (Figure 1). We demonstrate several examples, including energy materials, automotive applications and metal specimens (Figure 2), upon which the use of XRM and FIB/SEM information on the same specimen has contributed to a more complete understanding of a materials system.

In life sciences, correlative microscopy methods have existed for decades in various forms. Because it is fully acknowledged that no single microscopic imaging or characterization technique can provide a complete picture of a specimen [3], the challenge turns to finding practical methods of localizing the same feature in multiple microscopes. Most commonly, researchers have sought correlation between functional light microscopy information and ultrastructure in electron microscopy. XRM presents a new opportunity to bridge the length scales between the two and ease the 'needle in a haystack' navigation problem. One example where XRM is demonstrating its effectiveness as a correlative technique is in the field of neuroscience. There exists great interest in creating complete neural network maps of the brain and the need for information across multiple length scales in 3D has scale spawned the development of highthroughput 3D electron microscopy (3DEM) techniques, based on TEM and SEM methods incorporating either physical sectioning via cutting (ultra-microtome) or ion abrasion (FIB-SEM). However, these 3DEM techniques can only prosper if long acquisition times (or small sample volumes), sensitivity to sample preparation, and inefficiencies in locating regions of interest (buried subsurface features) can be averted. Recently, XRM techniques acting as a bridge between light- and electron-microscopy have acted as an efficiency multiplier in this domain (Figure 3). We conclude by offering perspectives on the future directions of the utilization of correlative microscopy techniques with respect to XRM information. 


\section{References}

[1] A. P. Merkle and J. Gelb, Ascent of 3D X-ray Microscopy in the Laboratory, Microscopy Today, 21 (2013), p. 10

[2] E Maire and P Withers, Quantitative X-ray tomography, International Materials Review, 59 (2014) p. 1

[3] S Handschuh, et al., Frontiers in Zoology, 10 (2013), p. 44
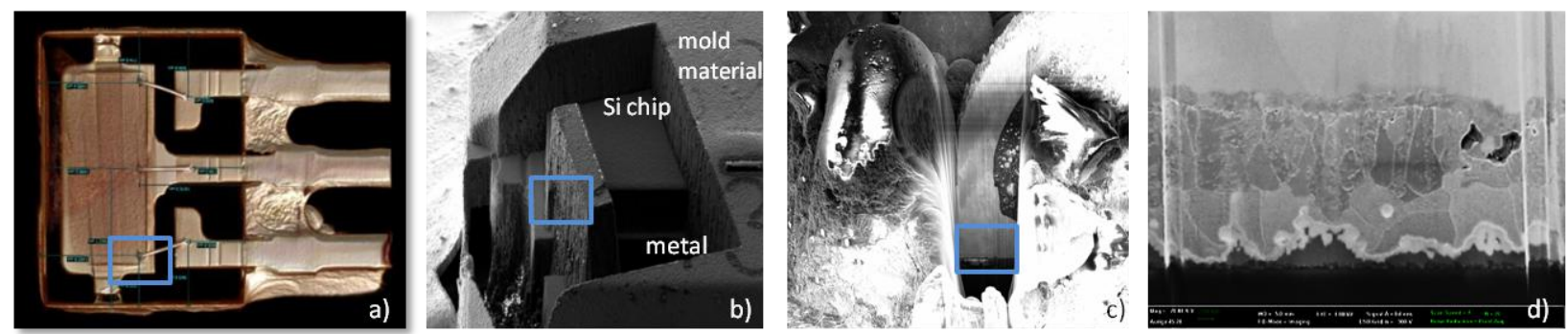

Figure 1. Target region navigation workflow, utilizing (a) XRM dataset to identify VOI, followed by (b-c) laser + FIB milling to quickly expose the feature and interface of interest on a Hall sensor device.

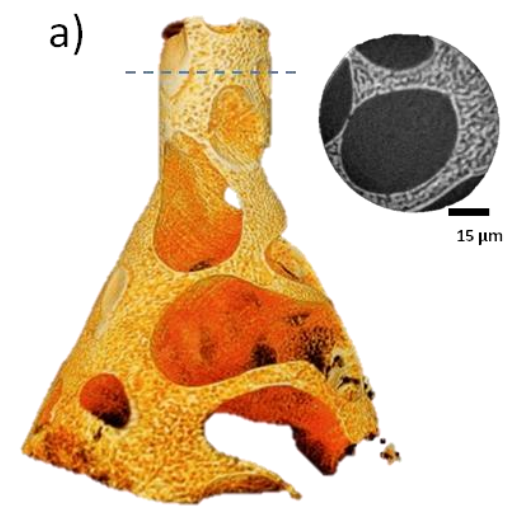

b)

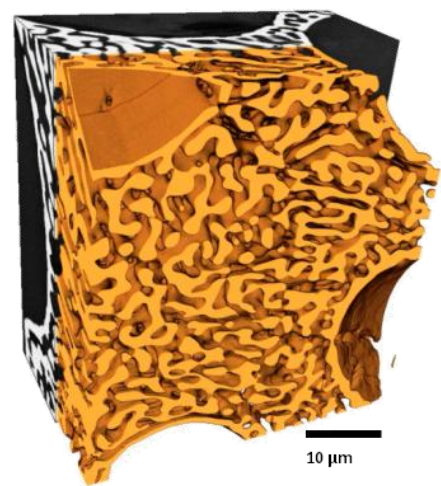

c)

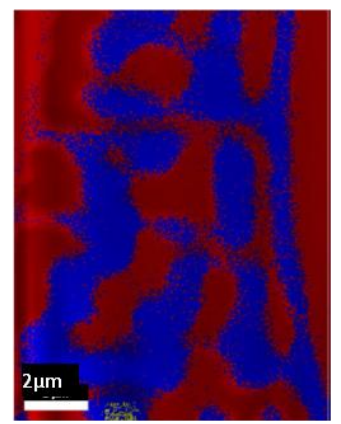

Figure 2. A single Aluminum Copper eutectic sample and volume-of-interest imaged a) nondestructively with XRM at multiple resolutions b) with FIB-SEM nanotomography including c) EDS for chemical information. Sample courtesy of B. Patterson, Los Alamos National Laboratory.

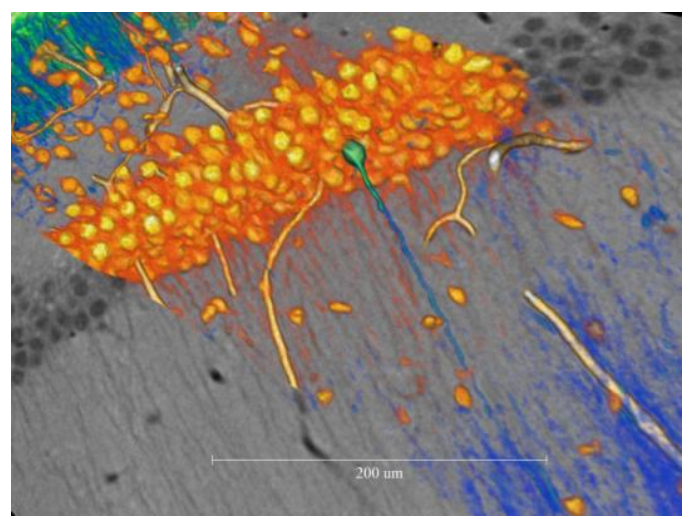

Figure 2. XRM dataset of stained (for EM) mammalian brain tissue. Such non-destructive XRM datasets are being used to navigate to specific subsurface volumes of interest quickly, thereby multiplying the efficiency of 3D EM techniques. Work is in collaboration with the National Center for Microscopy and Imaging Research (NCMIR) at UCSD together with Carl Zeiss X-ray Microscopy. 